\title{
An Analysis of the Differences in Business Students' Intercultural Sensitivity in two Degree Programmes
}

\author{
Ueberwimmer Margarethe, Hofstadler Hannes, Sophie Wiesinger \\ University of Applied Sciences Upper Austria, Austria
}

\begin{abstract}
The initial assessment and the in-between assessment of intercultural sensitivity of students of the University of Applied Sciences Upper Austria (UASUA) is examined, representing an important part of a planned longitudinal study. Additionally, the researchers present the design of the future. In general the researches want to explore the effects of intercultural education on business students. In this paper the researchers want to evaluate whether there is a difference in cultural sensitivity of students choosing two different business studies, one with, the other one without intercultural courses and inputs. Furthermore, the disparity between objective and subjective intercultural sensitivity is compared. In a next step the researchers analyze the effects of the first intercultural education part in one of the selected business studies. An approved test instrument (IDI) and an additional survey developed by the researchers are used for evaluation and t-tests are used to compare the two groups of students and the development of the sensitivity measurements over time. These findings contribute to a longitudinal study aimed at assessing the effectiveness of specific input on intercultural sensitivity over several generations of students.
\end{abstract}

\section{Introduction}

The researchers explore the effects of intercultural education on business students from a University of Applied Sciences in Austria. The research questions concerning the intercultural sensitivity of students are analyzed by comparing those who choose business studies with international and intercultural aspects and those who choose business studies without such specialized input. The motives and motivation for the choice of studies, sociodemographic data of the students, as well as data on their family background are collected and related to the results of a test instrument measuring intercultural sensitivity. Furthermore, the disparity between objective and subjective intercultural sensitivity is compared.

The study programme of Global Sales and Marketing (in this paper being referred to as GSM) at the University of Applied Sciences Upper Austria has only recently adapted its curriculum, introducing a modular set of intercultural lectures to the extent of 20 ECTS points. In comparison with other bachelor study programmes in Austria, this is an exceptional number of points, compared to any otherwise business focused programme.

Although both study programmes of which students are being compared are within the scope of business and management studies, GSM is different from the study programme Production and Management, for which in this article the abbreviation PMT will be used, in various aspects, with this special focus on intercultural education being the main difference. Students of the GSM programme are getting a profound introduction into cultural differences and similarities of incoming professors from all over the world and are taught one hundred percent in English, whereas PMT does not include any subjects of that kind. Instead, in the case of PMT, the focus is rather laid on education covering the areas of production and manufacturing, procurement, logistics, maintenance and quality management.

At this point, the concept of the semester abroad in both study programmes should also be highlighted. Whilst a semester abroad in the third semester is compulsory and one of the central elements of the GSM programme, PMT students have the possibility to also spend the third semester abroad but are not especially encouraged to do so.

Being a "fore-runner" among other higher educational institutions in Austria, it is of utmost importance, to evaluate and measure the impact of such a core curricula change.

Moreover, since intercultural investigations are becoming an increasingly important area in times of globalization, this paper attempts to provide a first insight into the assessment of effectiveness of intercultural education.

\section{Literature review}

\subsection{Steyr Intercultural Management Model}

The Steyr Intercultural Management Model (SIMM) has been developed at the University of Applied Sciences in Steyr. When comparing it to many cultural dimension approaches, it does not aim at just describing a culture, but finding out WHY a culture is the way it is, in order to derive knowledge of HOW to (inter-)act in this culture. For this purpose, the relationship of two cultures (one's own and a foreign one), which is understood as "cross- 
culture" in this model, should be investigated step by step on three levels.

On the first level, the macro level, both cultures are examined objectively in terms of their history, philosophy, religion, society, politics, economics and law. Subsequently, the results of these fields of research are compared and the two cultures' differences and similarities are elaborated. On the second level, subjective perceptions are compared with the objective results of the first level, with the purpose of demonstrating that the point of view, out of which a culture is seen, plays an important role when describing this culture. Finally, on the third level, the SIMM deals with individual influence factors like status, origin, education, environment, stereotypes or traditions, which are also included in the concept [12].

The SIMM (Steyr Intercultural Management Model) does not aim to clarify "HOW" cultures can be described, it aims at "WHY" cultures are the way they are, in order to then be able to derive from this knowledge basis "HOW" to (inter-)act [3, p. 10].

Considering the idea behind the SIMM, the intercultural course modules intend to reflect cultural backgrounds (e.g. historical, political, societal roots) and elaborate reasons for cultural differences, instead of concentrating too much on culture models and the usual cross-cultural theory [3, p.13].

\subsection{Intercultural sensitivity}

In order to clearly understand the IDI approach, it is necessary to firstly clarify exactly what is meant by the terms "cultural sensitivity" and "cultural competence”.

Hammer et al. use the term "intercultural sensitivity" to refer to the ability to discriminate and experience relevant cultural differences and use the term "intercultural competence" to mean the ability to think and act in intercultural appropriate ways [8, p. 422].

\subsection{Intercultural competence of business students}

In one of his articles, Cant talks about the importance of internationalizing the curriculum of business students in order to prepare them for the realities of global commerce, being able to take up global assignments. According to him, the need for globally competent managers is a response to the growth of global economy; however, American business students are still lacking in knowledge of other societies and are naive about the effect that cultural differences have on the conduct of international business. Therefore, international business studies need to form a normal part of the curriculum [4].
Similarly, Daly and Barker, investigating on the success of international student exchange programs, see a need for enhancing students' intercultural understanding and competence [6].

\subsection{The Intercultural Development Inventory}

The construction of the Intercultural Development Inventory (IDI) was based on the preliminary work undertaken by Bennett, who created the Developmental Model of Intercultural Sensitivity (DMIS), a framework for conceptualizing dimensions of intercultural sensitivity and competence [8, pp. 421-423].

When discussing intercultural sensitivity the most central author is Milton Bennett who set up a "Developmental Model of Intercultural Sensitivity" in 1993 which is the basis of the IDI test framework [1]. The six stages of intercultural sensitivity are as follows [2]:

1. Denial: Does not recognize cultural differences

2. Defence: Recognizes some differences, but sees them as negative

3. Minimization: Unaware of projection of own cultural values; sees own values as superior; treats everyone "equally"

4. Acceptance: Shifts perspectives to understand that the same "ordinary" behaviour can have different meanings in different cultures

5. Adaption: Can evaluate the behaviour of others from their frame of reference and can adapt behaviour to fit the norms of a different culture

6. Integration: Can shift frame of reference and also deal with resulting identity issues

Hammer et al. categorize these stages into ethnocentric and ethno-relative orientations by describing ethnocentric orientations as ways of "avoiding cultural difference" and ethno-relative worldviews as ways of "seeking cultural difference" [8].

\subsection{Other Studies}

Measuring the impact of social competence subjects is not a new approach. May et al. [11], for example, dealt with the effects of business ethics education on aspects like moral efficacy, moral meaningfulness and moral courage of business students from a major Midwestern university in the U.S. In a quasi-experimental pre-test-post-test design, a treatment and control group were compared and finally, the above named aspects were ranked.

In 2003, Dimitrov and Rumrill [7] published a paper, in which they examined issues involved in comparing groups and measuring change with pretest and post-test data. Similarly, the results of this study should compare the intercultural sensitivity of GSM and PMT students before and after their 
studies, bearing in mind that only GSM students had received intercultural education throughout their study program.

Pyne et al. reported findings from a longitudinal survey, where they collected opinions about "approaches which might enhance their intercultural competence" [13] of postgraduate logisticians at their point of enrolment and several months of work experience. The goal of their study is to test the importance of socio-cultural and language-related variables, comparing different years of the survey and nationality groups.

Several attempts have also been made to analyse new teaching approaches, teaching intercultural competence being one of them. In this context, a considerable amount of literature has been published on the integration of a semester abroad in study programs (e.g. [9], [6]). In comparison, this study additionally deals with the effects of providing students with profound intercultural knowledge on the enhancement of their cultural competence by applying the ideas of the SIMM.

In general, what is known about new teaching methods so far, is largely based upon empirical studies, such as that conducted by Cheng et al. [5], that investigate how new ways of learning effect students' results on tests and/or attitudes towards a subject.

The authors seek to extend these existing studies by exploring to what extent intercultural competence and sensitivity can be developed in an educational setting, thereby carrying out the first study of this kind among management students in Austria.

Table 1. Other studies

\begin{tabular}{l|l}
\hline Author & Research objective \\
\hline $\begin{array}{l}\text { Koskinen, Tossavainen } \\
\text { (2004) [9] }\end{array}$ & $\begin{array}{l}\text { Effects of semester abroad in study } \\
\text { programmes of nursing }\end{array}$ \\
\hline $\begin{array}{l}\text { Pyne, Dinwoodie, Roe } \\
\text { (2007) [13] }\end{array}$ & $\begin{array}{l}\text { Collection of opinions on approaches which } \\
\text { might enhance the intercultural competence } \\
\text { of postgraduate logisticians }\end{array}$ \\
\hline $\begin{array}{l}\text { Kai-Wen Cheng,Yu-Fen } \\
\text { Chen (2008)[5] }\end{array}$ & \begin{tabular}{l} 
Effects of cooperative learning \\
\hline $\begin{array}{l}\text { May, Luth, Schwoerer } \\
\text { (2009) [11] }\end{array}$
\end{tabular} \\
\hline Daly, Barker (2010) [6] & $\begin{array}{l}\text { Effects of business ethics education of U.S. } \\
\text { business students }\end{array}$ \\
\hline
\end{tabular}

\subsection{Linkage to concept of GSM study program}

In this respect, the GSM study program is aligned with the idea of developing intercultural sensitivity by moving through these six phases. The basis to overcome the first two stages is general interest in other cultures, which GSM students are supposed to bring along. This view is supported by Koskinen and Tossavinen who found out that students' intercultural desire, i.e. their effort to become intercultural competent turned out to be the foundation of the entire process of gaining intercultural competence [10]. Secondly, for moving from an ethno-centric to an ethno-relative worldview and for overcoming the stage of minimization, the SIMM is applied, providing students with the cultural knowledge necessary to thoroughly understand foreign cultures and experience one's own culture in the context of other cultures. In order to reach the stage of adaption, the semester abroad should help to include an affect on behaviour in addition to mere cognitive knowledge. Finally, it can be summarized that the study program GSM should prepare students for situations demanding intercultural competence such as long-term expatriation, in which many shift to the last stage, integration.

\section{Methodology}

The researchers focus on the following research questions when setting up the long-term research design.

RQ: Are differences in intercultural sensitivity and competence identifiable, when comparing students having chosen different types of business studies in Austria in the long run?

The following research questions are answered in the study of the first assessment [14].

RQ1: Are there differences in intercultural sensitivity recognizable of students having chosen different types of business studies?

RQ2: Are there differences in intercultural sensitivity recognizable according to the gender of the students?

RQ3: Are there differences in intercultural sensitivity when comparing regional aspects of students, i.e. whether the students come from urban or from rural areas?

RQ4: Are there differences in intercultural sensitivity when comparing students and their language background (e.g. mother tongue)?

Students of two chosen degree programmes (with and without an intercultural input) take an approved test instrument, the so-called intercultural development inventory (IDI) test, at the beginning of their studies, hence before the first contact with intercultural education. The IDI test consists of 50items presented in a five-point Likert-type scale, ranging from agree to disagree. Out of those items two scales are provided, the so-called 'overall perceived intercultural sensitivity' ('overall PIS'), a subjective measurement on cultural sensitivity, and the 'overall developmental intercultural sensitivity' ('overall DIS'), an objective measurement on cultural sensitivity. The difference between the subjective and objective measurement is calculated as the so-called 'orientation gap'. Furthermore, thirteen scales presented in figure 1 are calculated out of those items, i.e. 'denial cluster', 'disinterest in cultural difference', 'avoidance of interaction with 
cultural difference', 'defence cluster', 'reversal', 'minimization', 'similarity cluster', 'universalism cluster', 'acceptance cluster', 'adaption cluster', 'cognitive frame-shifting', 'behavioural codeshifting', and 'integration'. All scales are provided by IDI.

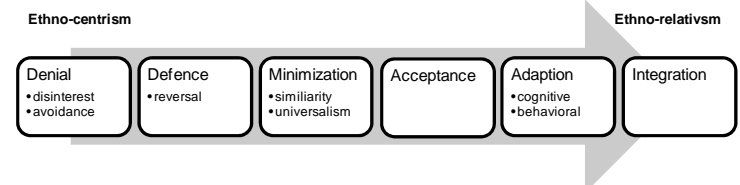

Figure 1. Dimensions of DMIS, adapted from [8]

An additional survey, developed by the researchers, supplements the first IDI testing by collecting socio-demographic data, e.g. migration background, family background and previous intercultural experience of the students.

The first (experimental) group consists of students taught by intercultural courses (GSM students), the second (control) group consists of students who receive a traditional business education without intercultural courses (PMT students). The numbers of the participants are 44 in the experimental group and 30 in the control group, 74 students in total. The testing of the students takes place before the courses start.

Using t-tests, possible differences between both groups of students are calculated and interpreted by comparing the means of various measurements on intercultural sensitivity.

Furthermore, the authors of this article analysed variables that could influence the effect of intercultural education, such as bilingual background, gender, area the students come from and mother tongue.

In the second assessment the researchers test whether there are effects in intercultural sensitivity after one year study in both considered groups of students. The research question is answered in the following.

RQ5: Are there differences in intercultural sensitivity recognizable of students having chosen different types of business studies after one year?

Using t-tests for paired samples, the effects of each group can be measured whereby again the first (experimental) group consists of students taught by intercultural courses, the second (control) group consists of students with a traditional business education without intercultural courses.

\section{Hypotheses of First Assessment}

Based on research question RQ1, the following hypotheses can be defined, asking for differences in cultural sensitivity, comparing the experimental group (GSM students) and the control group (PMT students):
H1: There is no difference in cultural sensitivity between the experimental group (GSM students) and the control group (PMT students) at the beginning of the study programme.

H1a: There is no difference in cultural sensitivity between the experimental group (GSM students) and the control group (PMT students) considering only the male students.

H1b: There is no difference in cultural sensitivity between the experimental group (GSM students) and the control group (PMT students) considering only the female students.

RQ2 leads to the hypotheses on differences in cultural sensitivity comparing the gender of the students.

H2: There is no difference in cultural sensitivity between male and female students at the beginning of the study programme.

H2a: There is no difference in cultural sensitivity between male and female GSM students.

H2b: There is no difference in cultural sensitivity between male and female PMT students.

RQ3 leads to the hypotheses on differences in cultural sensitivity comparing regional aspects of students, based on whether they come from urban or from rural areas.

H3: There is no difference in cultural sensitivity between students coming from a rural and an urban area at the beginning of the study programme.

H3a: There is no difference between GSM students coming from a rural and an urban area.

H3b: There is no difference between PMT students coming from a rural and an urban area.

RQ4 leads to the hypotheses on differences in cultural sensitivity comparing students and their language background.

H4a: There is no difference in cultural sensitivity between students having German as their mother tongue and having another mother tongue.

H4b: There is no difference in cultural sensitivity between students being monolingual and multilingual (considering the mother tongue).

\section{Findings and Results of First Assessment}

The results of the descriptive statistics comparing the students in the experimental group and in the control group are shown in table 2 whereby 'Mean' is the average value of the considered group, 'Std' denotes the standard deviation, and ' $\mathrm{N}$ ' is the number of persons in the group. 
Table 2. Descriptive statistical analyses between the two groups

\begin{tabular}{lrr|rr} 
& $\begin{array}{r}\text { Experimental group } \\
\text { GSM }\end{array}$ & \multicolumn{2}{|c}{$\begin{array}{c}\text { Control group } \\
\text { PMT }\end{array}$} \\
& $\begin{array}{l}\text { Mean } \\
\text { Scale }\end{array}$ & Std & Mean & Std \\
\hline overall PIS & 119,85 & 4,25 & 116,88 & 4,68 \\
overall DIS & 87,71 & 11,60 & 83,22 & 13,77 \\
orientation gap & 32,15 & 7,83 & 33,66 & 9,40 \\
\hline denial cluster & 3,89 & 0,52 & 3,61 & 0,68 \\
disinterest & 3,76 & 0,64 & 3,38 & 0,77 \\
avoidance & 4,08 & 0,60 & 3,92 & 0,78 \\
\hline defence cluster & 3,92 & 0,61 & 3,67 & 0,77 \\
reversal & 3,47 & 0,58 & 3,60 & 0,70 \\
\hline minimization & 2,75 & 0,55 & 2,67 & 0,44 \\
similarity cluster & 2,61 & 0,77 & 2,55 & 0,68 \\
universalism cluster & 2,93 & 0,59 & 2,82 & 0,71 \\
\hline acceptance cluster & 3,83 & 0,68 & 3,29 & 0,77 \\
\hline adaption cluster & 3,33 & 0,63 & 2,89 & 0,59 \\
cognitive frame-shifting & 3,11 & 0,83 & 2,70 & 0,71 \\
behavioural code-shifting & 3,50 & 0,59 & 3,04 & 0,76 \\
\hline integration & 4,15 & 0,71 & 3,75 & 0,76 \\
\hline
\end{tabular}

To decide whether the scales of the GSM students are equal to those of the PMT students, t-tests for each scale are performed. Table 3 says that there is a significant difference in the overall perceived intercultural sensitivity, the subjective measurement on intercultural sensitivity, comparing GSM and PMT students. Although there is a difference in the ethno-relativism scales, i.e. acceptance, adaption and integration, the overall development intercultural sensitivity scale, the overall objective measurement, of both groups, is said to be equal. It has to be concluded that hypothesis $\mathrm{H} 1$ has to be rejected. This means that the students who study the GSM programme with an intercultural aspect tend to be more intercultural sensitive than students who study another programme without intercultural aspects.

Table 3. Inductive statistical analyses

\begin{tabular}{lcc} 
Scale & \multicolumn{2}{c}{ t-test $\mathrm{p}$-value (2-sided) } \\
\hline overall PIS & 2,835 & $0,006 * *$ \\
overall DIS & 1,515 & 0,134 \\
orientation gap & $-0,755$ & 0,452 \\
\hline denial cluster & 1,988 & $0,051 *$ \\
disinterest & 2,255 & $0,027 * *$ \\
avoidance & 0,914 & 0,365 \\
\hline defence cluster & 1,52 & 0,133 \\
reversal & $-0,841$ & 0,403 \\
\hline minimization & 0,679 & 0,500 \\
similarity cluster & 0,322 & 0,749 \\
universalism cluster & 0,758 & 0,451 \\
\hline acceptance cluster & 3,165 & $0,002 * *$ \\
\hline adaption cluster & 3,024 & $0,003 * *$ \\
cognitive frame-shifting & 2,229 & $0,029 * *$ \\
behavioural code-shifting & 2,929 & $0,005 * *$ \\
\hline integration & 2,297 & $0,025 * *$ \\
\hline & \multicolumn{2}{c}{$* * \mathrm{p}<0,05 ; * \mathrm{p}<0,1$}
\end{tabular}

Comparing the male students of both groups - the 20 male GSM students and the 21 male PMT students - one can see that there is a significant difference in both overall intercultural sensitivity measurements resulting from significant differences in all measurements except the minimization measurements. Therefore hypothesis $\mathrm{H} 1 \mathrm{a}$ has to be rejected as well.

Comparing the female students of both groups the 24 female GSM students and the 9 female PMT students - one can see that there is a significant difference in the overall development in the intercultural sensitivity measurement $(p<0,1)$ resulting from significant differences in measurements of the defence and adaption scale. The orientation gap differs significantly $(p<0,05)$. Therefore, hypothesis H1b has to be rejected.

To answer research question RQ2, the whole sample of students has to be analysed according to the gender of the students. The descriptive analysis and the corresponding t-tests are shown in table 4 . The subjective 'overall perceived intercultural sensitivity' of the 33 female students is significantly $(p<0,1)$ higher than the subjective sensitivity of the 41 male students. The denial cluster $(p<0,05)$ and the defence cluster $(\mathrm{p}<0,1)$ differ as well. All other intercultural sensitivity measurements can be said to be equal. The objective 'overall developmental intercultural sensitivity' of both groups does not differ significantly. Therefore hypothesis $\mathrm{H} 2$ has to be accepted as a difference in intercultural sensitivity and cannot be detected when comparing male and female students.

Comparing the 20 male students and the 24 female students of the GSM study programme, one can see that there is no significant difference in any intercultural sensitivity measurement. Therefore, hypothesis H2a has to be accepted as well.

Comparing the 21 male students and the 9 female students of the PMT study programme, one can see that there are significant differences in intercultural sensitivity, especially in both overall measurements, in the denial, defence and integration measurements. Therefore, hypothesis H2b has to be rejected.

Table 4. Analyses of male and female students

\begin{tabular}{lrr|rrrr}
\multicolumn{2}{c|}{$\begin{array}{c}\text { male } \\
(\mathrm{N}=41)\end{array}$} & \multicolumn{2}{c}{$\begin{array}{c}\text { female } \\
(\mathrm{N}=33)\end{array}$} & & \\
Scale & Mean & Std & Mean & Std & t-test & $\mathrm{p}$-value \\
\hline overall PIS & 117,79 & 5,02 & 119,71 & 3,94 & $-1,800$ & $0,076 *$ \\
overall DIS & 83,90 & 13,63 & 88,36 & 10,98 & $-1,522$ & 0,132 \\
orientation gap & 33,89 & 9,15 & 31,36 & 7,45 & 1,284 & 0,203 \\
\hline \multicolumn{7}{c}{}
\end{tabular}

To answer research question RQ3, the whole sample of students has to be analysed accordingly, as to whether the students come from an urban or a rural area. None of the sensitivity measurements of both groups differs significantly. Therefore, hypothesis H3 has to be accepted as a difference in intercultural sensitivity cannot be detected when comparing the 51 students coming from a rural area and the 23 students coming from an urban area. The same holds for a comparison of rural and urban 
students, if the GSM students respectively the PMT students, are analyzed separately. Hence, hypothesis H3a and H3b have to be accepted.

To answer research question RQ4, the whole sample of students has to be analysed accordingly, to the mother tongue of the students. Most students come from Austria and Germany. Therefore, the item is simplified to a dichotomous variable asking whether the mother tongue is German or not. None of the sensitivity measurements of both groups differs significantly, except 'cognitive frameshifting'. Therefore, hypothesis $\mathrm{H} 4 \mathrm{a}$ has to be accepted: a difference in intercultural sensitivity cannot be detected comparing the 67 students with a mother tongue of German and the 7 students with another mother tongue.

To answer research question RQ4, the whole sample of students has to be analysed accordingly to the language background of the students. The item is simplified to a dichotomous variable indicating a monolingual (one mother tongue) or a multilingual (two or more mother tongues) background of the students. None of the sensitivity measurements of both groups differs significantly. Therefore, hypothesis H4b has to be accepted: a difference in intercultural sensitivity cannot be detected when comparing the 70 students with monolingual background and the 3 students with a multilingual background.

\section{Hypotheses of the Second (In-between) Assessment}

Based on research question RQ5, the following hypotheses can be defined, asking for differences in cultural sensitivity, comparing the experimental group (GSM students) and the control group (PMT students):

H5: There is no difference in cultural sensitivity between the experimental group (GSM students) and the control group (PMT students) after one year, after the intercultural education of the experimental group.

H5a: There is no difference in cultural sensitivity in the experimental group (GSM students) after one year, after the intercultural education.

H5b: There is no difference in cultural sensitivity in the control group (PMT students) after one year.

\section{Findings and Results of the Second (In- between) Assessment}

At the second critical time point i.e. after the theoretical intercultural input (one year after beginning their studies), the intercultural development inventory (IDI) test is conducted again. At the first assessment 44 GSM students and 30 PMT students took part in the study. Thereof 42
GSM students and 19 PMT students took part at the second time point of measurement.

To answer research question RQ5 t-tests of paired samples and a regression analysis are performed. Ttests of paired samples give an answer to changes of intercultural sensitivity over the time when analyzing the GSM or the PMT students separately.

However, not all students of the first assessment take part in the second assessment, therefor the sample shrinks - in total there are 42 GSM students and 19 PMT students who can be compared.

The descriptive analysis of the 42 GSM students and the corresponding t-tests of the paired samples are shown in table 5. Although the overall measurements are not significant $(p>0,1)$, there is an indication of effects in the cluster measures minimization, acceptance and adaption (all $\mathrm{p}<0,05$ ), a shift towards more ethno-relativism of the students is recognizable. These findings have to be proofed for a larger sample of students, a longitudinal study of these effects is necessary.

Table 5. Statistics comparing GSM students after one

\begin{tabular}{lrrrr}
\multicolumn{4}{c}{ year } \\
& \multicolumn{4}{c}{$\begin{array}{c}\text { GSM } \\
\text { (N=42) }\end{array}$} \\
Scale & Mean & Std & t-test p-value (2-sided) \\
\hline overall PIS & 0,94 & 4,71 & 1,296 & 0,202 \\
overall DIS & 1,16 & 11,55 & 0,650 & 0,519 \\
orientation gap & $-0,17$ & 7,19 & $-0,152$ & 0,880 \\
\hline denial cluster & 0,05 & 0,58 & 0,609 & 0,546 \\
disinterest & 0,02 & 0,72 & 0,214 & 0,831 \\
avoidance & $-0,02$ & 0,78 & $-0,200$ & 0,843 \\
\hline defence cluster & 0,05 & 0,69 & 0,489 & 0,627 \\
reversal & 0,05 & 0,58 & 0,591 & 0,557 \\
\hline minimization & $-0,17$ & 0,59 & $-1,824$ & $0,075 *$ \\
similarity cluster & $-0,31$ & 0,83 & $-2,468$ & $0,018 * *$ \\
universalism cluster & $-0,13$ & 0,82 & $-0,991$ & 0,328 \\
\hline acceptance cluster & 0,31 & 0,86 & 2,374 & $0,022 * *$ \\
\hline adaption cluster & 0,22 & 0,62 & 2,306 & $0,026 * *$ \\
cognitive frame-shifting & 0,43 & 0,75 & 3,749 & $0,001 * *$ \\
behavioural code-shifting & 0,21 & 0,70 & 1,952 & $0,058 *$ \\
\hline integration & $-0,05$ & 0,71 & $-0,432$ & 0,668 \\
\hline
\end{tabular}

The descriptive analysis of the 19 PMT students and the corresponding t-tests of the paired samples are shown in table 6. Nearly none of the measurements is significant $(p>0,1)$, only the measurement for disinterest is significant $(p<0,1)$ indicating a small increase in the disinterest of PMT students. Again, these findings have to be proofed for a larger sample of students. 
Table 6. Statistics comparing PMT students after one year

\begin{tabular}{|c|c|c|c|c|}
\hline \multirow[b]{2}{*}{ Scale } & \multicolumn{4}{|c|}{$\begin{array}{c}\text { Control group } \\
\text { PMT } \\
(\mathrm{N}=19)\end{array}$} \\
\hline & Mean & Std & t-test & lue (2-sided) \\
\hline overall PIS & $-0,38$ & 4,50 & $-0,364$ & 0,720 \\
\hline overall DIS & 0,07 & 9,95 & 0,031 & 0,976 \\
\hline orientation gap & $-0,45$ & 5,88 & $-0,330$ & 0,745 \\
\hline denial cluster & 0,12 & 0,49 & 1,070 & 0,299 \\
\hline disinterest & 0,25 & 0,55 & 1,971 & $0,064 * *$ \\
\hline avoidance & $-0,05$ & 0,70 & $-0,323$ & 0,750 \\
\hline defence cluster & 0,11 & 0,38 & 1,205 & 0,244 \\
\hline reversal & $-0,02$ & 0,49 & $-0,215$ & 0,832 \\
\hline minimization & $-0,08$ & 0,64 & $-0,515$ & 0,613 \\
\hline similarity cluster & $-0,06$ & 0,87 & $-0,315$ & 0,757 \\
\hline univers alism cluster & $-0,09$ & 0,71 & $-0,563$ & 0,580 \\
\hline acceptance cluster & $-0,13$ & 0,52 & $-1,064$ & 0,301 \\
\hline adaption cluster & $-0,09$ & 0,47 & $-0,809$ & 0,429 \\
\hline cognitive frame-shifting & 0,03 & 0,56 & 0,203 & 0,841 \\
\hline behavioural code-shifting & $-0,18$ & 0,60 & $-1,309$ & 0,207 \\
\hline integration & 0,07 & 0,73 & 0,441 & 0,664 \\
\hline
\end{tabular}

Furthermore regression models are estimated to identify the effect of being a student of the GSM programme and hence of having an intercultural education. Our approach is to identify linear regression models of the form

$$
y_{2, i}=c+\alpha \cdot y_{1, i}+\beta \cdot d+\epsilon_{i}
$$

where $i$ is the number of the student, $y_{2, i}$ is the considered measurement of the given student at the in-between assessment, $y_{1, i}$ is the considered measurement of the given student at the first assessment, $d$ a dummy variable indicating whether the student is in the experimental group $(d=1)$ or in the control group $(d=0)$ and $\epsilon_{i}$ is the error. To estimate the 'overall perceived intercultural sensitivity' (overall PIS) the following model is identified

$$
\hat{y}_{2, i}=57,573+0,504 \cdot y_{1, i}+2,585 \cdot d
$$

where $y_{1, i}$ is the overall PIS of the given student at the first assessment. All parameters are significant (p-value < 0.05) and $R^{2}=0,561$. The model indicates a positive effect of the GSM study on the overall PIS due to the positive parameter of the dummy variable. Therefore H5 can be accepted.

\section{Conclusion}

The results of the study demonstrate that one can say the intercultural sensitivity of students having chosen a study programme with an intercultural education differs in comparison to students having chosen a classical study programme. This holds for male as well as for female students, whereby the gap of various intercultural sensitivity measurements between male students is higher and has a tendency to be significantly higher.

Regarding all students of this study, there are no significant differences in intercultural sensitivity if one analyses the male versus the female students, especially if one has a closer look at the GSM students. However, there are gender differences if one analyzes the PMT students.

Some other possible relevant influencing factors are analyzes, i.e. the area - urban or rural area where the students come from; mother tongue of the students; language background. None of those characteristics has an effect on the intercultural sensitivity of students, neither for the whole group nor for GSM respectively PMT students solely.

These findings contribute to a longitudinal study aimed at assessing the effectiveness of specific input on intercultural sensitivity over several generations of students. The next steps are as follows:

1. One question that needs to be asked in this context is whether significant changes in students' development of intercultural sensitivity will be detected after having spent the semester abroad. The authors expect more improvement of GSM students on the post-test results compared to control group (PMT). Thereby, a quasi-experimental study will follow a pre-test-post-test design, in which one experimental group (GSM students) is exposed to a treatment (intercultural education) and then compared to one control group (PMT students) who did not receive the treatment. At the third critical time point i.e. after a semester abroad (two years after beginning their studies), the intercultural development inventory (IDI) test will be conducted again.

2. Time-based changes in intercultural sensitivity can be already identified in the GSM students' group although the sample is rather small. Therefore this quasi-experimental study can be enlarged to a longitudinal study, so that more agegroups of students can be compared. The authors hope to get a larger database and to recognize longitudinal changes in intercultural sensitivity.

3. It is planned to invite business courses with a similar curriculum to GSM in Austria and Europe to participate in this study to get a more "global" comparison of the effects of intercultural education as well.

The next steps as described above are demonstrated in figure 2. 


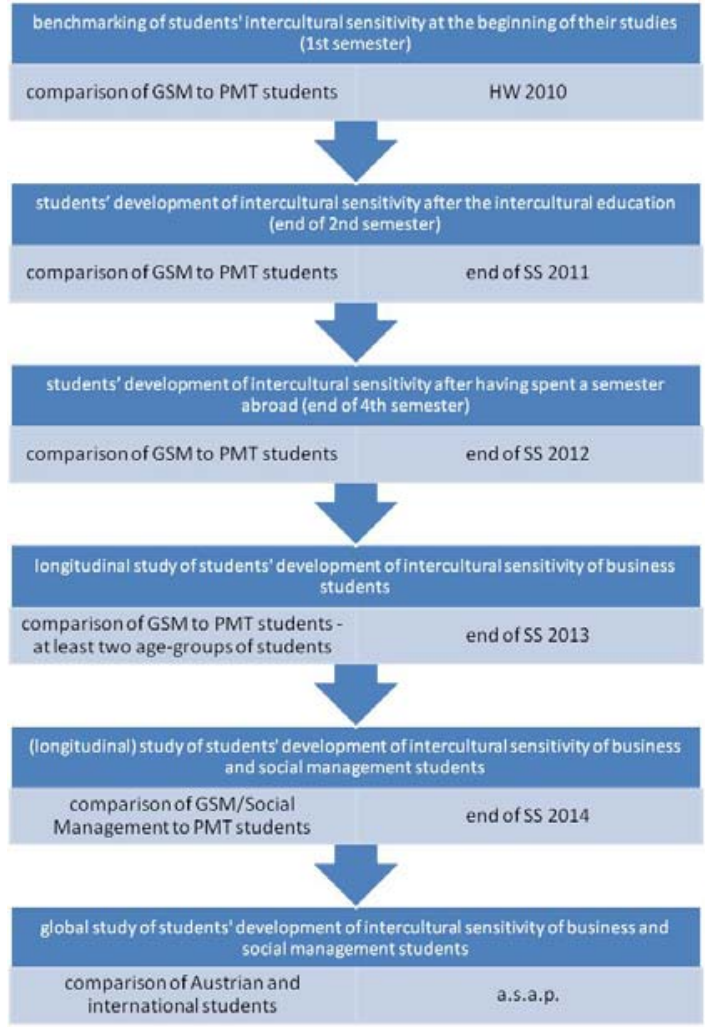

Figure 2. Steps of longitudinal study [15]

\section{References}

[1] M.J. Bennett, "Towards Ethnorelativism: A Developmental Model of Intercultural Sensitivity”, In R. M. Paige (Ed.), Education for the intercultural experience, Yarmouth, ME: Intercultural Press, 1993, pp. 21-72.

[2] D. Blackwell, "Cultural Competence”, Medical Marketing \& Media 40 (4), 2005, pp. 52-56.

[3] I. Boitllehner, H. Hofstadler, "Cultural Background Knowledge and Self-Reflection - is this enough to avoid cross-cultural Conflicts?“, Understanding Conflicts: Cross-Cultural Perspectives, Aarhus, Dänemark, 2008.

[4] A. G. Cant, "Internationalizing the Business Curriculum: Developing Intercultural Competence”, Journal of American Academy of Business, Cambridge 5 (1/2), 2004, pp. 177-182.

[5] K. Cheng, Y. Chen, "Effects of Cooperative Learning in a College Course on Student Attitudes toward Accounting: A Quasi-Experimental Study”, International Journal of Management 25 (1), 2008, pp. 111-118.

[6] A. Daly, M. Barker, “Australian universities' strategic goals of student exchange and participation rates in outbound exchange programmes", Journal of Higher Education Policy \& Management 32 (4), 2010, pp. 333342.

[7] D.M. Dimitrov, P.D. Rumrill, "Pretest-posttest designs and measurement of change”, Work 20 (2), 2003, pp. 159164.
[8] M.R. Hammer, M.J. Bennett, R. Wiseman, "Measuring intercultural sensitivity: The intercultural development inventory", International Journal of Intercultural Relations 27 (4), 2003, pp. 421-443.

[9] L. Koskinen, K. Tossavainen, "Study abroad as a process of learning intercultural competence in nursing”, INT J NURS PRACT 10 (3), 2004, pp. 111-120.

[10] L. Koskinen, K. Tossavainen, "Intercultural nursing. Benefits/problems of enhancing students' intercultural competence”, BR J NURS 12 (6), 2003, pp. 369-377.

[11] D.R. May, M. Luth, C.E. Schwoerer, “The effects of business ethics education on moral efficacy, moral meaningfulness, and moral courage: a quasi-experimental study”, Academy of Management Annual Meeting Proceedings, 2009, pp. 1-6.

[12] I. Mayer, H. Hofstadler, "Background Knowledge and Self-Reflection - a Prerequisite for Successful Cross Cultural Communication?", Conference Paper for the WCPHE-Conference 2007 in Monterrey, Mexico, 2007.

[13] R. Pyne, J. Dinwoodie, M. Roe, "Enhancing the intercultural competence of postgraduate logisticians", International Journal of Logistics: Research \& Applications 10 (3), 2007, pp. 221-233.

[14] M. Ueberwimmer, H. Hofstadler, S. Wiesinger, "An Analysis of the Differences in Business Students' Intercultural Sensitivity in two Degree Programmes”, In: Charles Shoniregun, Proceedings of the International Ireland Conference on Education - IICE-2012, ISBN 9781-908320-06-3, Dublin, Ireland, 2012, pp. 67-73.

[15] M. Ueberwimmer, B. Jociz, H. Hofstadler, "Design of a Study of Intercultural Sensitivity in the Choice of Studies of Business Students”, In: R. Füreder et al., Proceedings Cross-Cultural Conference 2012, ISBN 978-3-8440-09880, Shaker Verlag, 2012, p.47-53. 